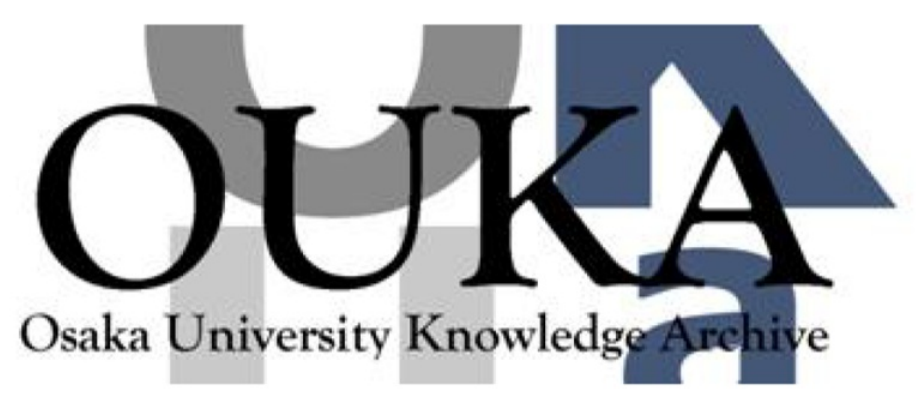

\begin{tabular}{|c|l|}
\hline Title & $\begin{array}{l}\text { Logarithmic Hodge Structures and Classifying } \\
\text { Spaces }\end{array}$ \\
\hline Author(s) & Kato, Kazuya; Usui, Sampei \\
\hline Citation & $\begin{array}{l}\text { The Arithmet ic and geometry of algebraic cycles } \\
\text { proceedings of the CRM summer school, June 7- } \\
19,1998, \text { Banff, Alberta, Canada. p. 115-p. 130 }\end{array}$ \\
\hline Issue Date & 2000 \\
\hline oaire:version & AM \\
\hline URL & https://hdl. handle. net/11094/73421 \\
\hline rights & $\begin{array}{l}\text { ○ 2000 by the American Mathematical Society. } \\
\text { All rights reserved. }\end{array}$ \\
\hline Note & \\
\hline
\end{tabular}

Osaka University Knowledge Archive : OUKA

https://ir. Library. osaka-u. ac. jp/

Osaka University 


\title{
LOGARITHMIC HODGE STRUCTURES AND CLASSIFYING SPACES
}

\author{
KaZuya Kato, SAmpei Usui
}

\section{INTRODUCTION}

This work is an attempt to add 'points at infinity' to the classifying space of polarized Hodge structures (PH, for short) of arbitrary weight. It is based on the following two ideas:

(0.1) We introduce the notion of polarized logarithmic Hodge structures (PLH, for short) ( $\$ 5$ ). Polarized logarithmic Hodge structures are defined by using the theory of logarithmic structures of Fontaine-Illusie (cf. [I], [K1]), and they work well in the analysis of degenerations of polarized Hodge structures. Our principle is that we enlarge the classifying space of polarized Hodge structures in the following way (for precise definitions, see Definitions in $(2.1),(5.2))$ :

$$
\begin{aligned}
& D:=(\text { Classifying space of } \mathrm{PH}) \subset \\
& D_{\Sigma}:=(\text { Space of nilpotent orbits in directions in } \Sigma), \\
& \Gamma \backslash D_{\Sigma}=(\text { Classifying space of PLH with local monodromies } \\
& \quad \text { in directions in } \Sigma \text { and with global monodromy } \Gamma) .
\end{aligned}
$$

Here $\Gamma$ is a discrete subgroup of $\operatorname{Aut}(D)$ satisfying certain conditions.

(0.2) We introduce the notions of generalized analytic spaces and generalized $f_{s}$ logarithmic analytic spaces (see (4.2)). Our extended space $\Gamma \backslash D_{\Sigma} \supset \Gamma \backslash D$ is not in general an analytic space. For instace, it can be something like

$$
S:=\left\{(x, y) \in \mathbf{C}^{2} ; x \neq 0\right\} \cup\{(0,0)\} .
$$

The space $\Gamma \backslash D_{\Sigma}$ and the above space $S$ admit the structure of generalized analytic space or even generalized fs logarithmic analytic space. In order to give $S$ the structure of generalized analytic space, the essential point is to endow it with the so-called strong topology (see (4.1)), which is strictly stronger than the topology as a subspace of $\mathbf{C}^{2}$.

Our main result says that the generalized fs logarithmic analytic space $\Gamma \backslash D_{\Sigma}$ is a fine moduli space of polarized logarithmic Hodge structures (§6). In a way, this realizes one of the dreams of Griffiths in [G].

We would like to add a remark. $D$ is always a homogeneous complex manifold. Assume that the horizontal tangent bundle of $D$ is not trivial. Then, it is a Hermitian symmetric domain if and only if the set of Hodge numbers is one of the following three cases:

(i) $w=2 t+1, h^{p, q}=0$ unless $p=t+1, t$.

(ii) $w=2 t, h^{p, q}=1$ for $p=t+1, t-1, h^{t, t}$ is arbitrary, $h^{p, q}=0$ otherwise.

(iii) $w=2 t, h^{p, q}=1$ for $p=t+a, t+a-1, t-a+1, t-a$ for some $a \geq 2, h^{p, q}=0$ otherwise.

Familiar (analytic) compactifications of $\Gamma \backslash D$ such as the Satake-Baily-Borel, the Borel-Serre, and toroidal are constructed only for those $D$ which are Hermitian symmetric domains. The only partially successful attempts to go beyond the symmetric case are the work [CK] of Cattani-Kaplan and the work [U2]. By the construction of the present article, we can talk about, for example, the extended period mapping associated to a degeneration of surfaces of general type, or of Calabi-Yau manifolds. One of the remaining

1991 Mathematics Subject Classification. Primary 14C30; Secondary 14D07, 32G20. Partly supported by the Grantsin-Aid for Scientific Research (1) (B): 08304002, the Ministry of Education, Science and Culture, Japan 
open problems is to find 'big fans' $\Sigma$ so that the spaces $\Gamma \backslash D_{\Sigma}$ contain all possible boundary points. This work is a continuation of the study [U2], which treats the part of $\Gamma \backslash D_{\Sigma}$, and is also a continuation of the study $[\mathrm{KkNc}]$ on logarithmic complex geometry.

This article is an abbreviated account. A paper [KU] with complete proofs will appear elsewhere.

The authors are grateful to Professor Kazuhiro Fujiwara for stimulating discussions. They are grateful to Professor Steven Zucker for his careful reading of the earlier versions of this paper, and for his valuable suggestions and comments on presentation. They are also grateful to the organizers of NATO ASI/1998 CRM Summer School at Banff, Canada.

\section{Contents}

$\S 1$. Preliminaries

$\S 2$. Spaces of nilpotent orbits

$\S 3$. Spaces of nilpotent $i$-orbits

$\S 4$. Topology, Generalized fs logarithmic analytic spaces

$\S 5$. Polarized logarithmic Hodge structures

$\S 6$. Main result

$\S 7$. Examples

\section{§1. Preliminaries}

(1.1) Classifying spaces of Hodge structures.

Let $\left(h^{p, q}\right)_{p+q=w}$, with $h^{p, q}=h^{q, p}$, be a set of Hodge numbers of weight $w$. Let $H_{0}$ be a free $\mathbf{Z}$-module of rank $\sum h^{p, q}$ and let $\langle,\rangle_{0}$ be a non-degenerate $\mathbf{Q}$-bilinear form on $H_{0, \mathbf{Q}}:=\mathbf{Q} \otimes H_{0}$ which is symmetric if $w$ is even and anti-symmetric if $w$ is odd. We denote by $D$ the set of all decreasing filtrations $F$ on $H_{0, \mathbf{C}}:=\mathbf{C} \otimes H_{0}$ such that $\left(H_{0},\langle,\rangle_{0}, F\right)$ is a polarized Hodge structure of Hodge type $\left(h^{p, q}\right)_{p+q=w}$. We denote by $\check{D}$ the set of all decreasing filtrations $F$ on $H_{0, \mathbf{C}}$ such that $\operatorname{dim} F^{p}=\sum_{r \geq p} h^{r, w-r}$ and $\left\langle F^{p}, F^{q}\right\rangle_{0}=0$ for $p+q>w$.

Let $G_{\mathbf{Z}}:=\operatorname{Aut}\left(H_{0},\langle,\rangle_{0}\right)$. For $A=\mathbf{Q}, \mathbf{R}, \mathbf{C}$, we denote $G_{A}:=\operatorname{Aut}\left(H_{0, A},\langle,\rangle_{0}\right)$ and $\mathfrak{g}_{A}:=\operatorname{Lie} G_{A}$, Then $\check{D}$ is a compact homogeneous space under $G_{\mathbf{C}}, D$ is an open subset of $\check{D}$ that is homogeneous under $G_{\mathbf{R}}$. Let $\Gamma$ be a subgroup of $G_{\mathbf{Z}}$. Then $\Gamma \backslash D$ is the classifying space of polarized Hodge structures on $H_{0}$ with the given Hodge numbers $\left(h^{p, q}\right)_{p+q=w}$ and with the given global monodromy $\Gamma$.

(1.2) Fans.

Definitions. (1.2.1) A subset $\sigma \subset \mathfrak{g}_{\mathbf{Q}}$ is a nilpotent cone if it satisfies the following two conditions:

(i) $\sigma$ is a finitely generated cone in $\mathfrak{g}_{\mathbf{Q}}$ consisiting of mutually commuting nilpotent elements.

(ii) $\sigma \cap(-\sigma)=\{0\}$.

(1.2.2) $A$ fan in $\mathfrak{g}_{\mathbf{Q}}$ is a non-empty set $\Sigma$ of nilpotent cones in $\mathfrak{g}_{\mathbf{Q}}$ satisfying the following two conditions:

(i) If $\sigma \in \Sigma$ then any face of $\sigma$ belongs to $\Sigma$.

(ii) If $\sigma, \sigma^{\prime} \in \Sigma$ then $\sigma \cap \sigma^{\prime}$ is a face of $\sigma$ and of $\sigma^{\prime}$.

(1.2.3) A subgroup $\Gamma \subset G_{\mathbf{Z}}$ is strongly compatible with a fan $\Sigma$ in $\mathfrak{g}_{\mathbf{Q}}$ if it satisfies the following two conditions:

(i) If $\gamma \in \Gamma$ and $\sigma \in \Sigma$ then $\gamma \sigma \gamma^{-1} \in \Sigma$.

(ii) If $\sigma \in \Sigma$ and if we define

$$
\Gamma(\sigma):=\{\gamma \in \Gamma ; \gamma: \text { unipotent, } \log \gamma \in \sigma\},
$$

then $\log \Gamma(\sigma)$ generates the cone $\sigma$.

Example. Let

$$
\Xi:=\left\{\sigma ; \sigma \text { is a nilpotent cone in } \mathfrak{g}_{\mathbf{Q}}, \operatorname{rank}(\sigma) \leq 1\right\} .
$$

Then $\Xi$ is a fan. For any subgroup $\Gamma$ of $G_{\mathbf{Z}}$ with finite index, $\Gamma$ is automatically strongly compatible with $\Xi$. Whereas, $\Gamma=\{1\}$ is not strongly compatible with $\Xi$, i.e., the condition (1.2.3) (ii) is not fulfiled.

(1.3) Nilpotent orbits and nilpotent $i$-orbits. 
Definition. Let $\sigma$ be a nilpotent cone in $\mathfrak{g}_{\mathbf{Q}}$. For $A=\mathbf{R}, \mathbf{C}$, we denote by $\sigma_{A}$ the A-linear span of $\sigma$ in $\mathfrak{g}_{A}$. A subset $Z$ of $\check{D}$ is a $\sigma$-nilpotent orbit (resp. $\sigma$-nilpotent $i$-orbit) if it satisfies the following three conditions for some $F \in Z$.

(i) $Z=\exp \left(\sigma_{\mathbf{C}}\right) F\left(\right.$ resp. $\left.Z=\exp \left(i \sigma_{\mathbf{R}}\right) F\right)$.

(ii) $N F^{p} \subset F^{p-1}(\forall p, \forall N \in \sigma)$, (Griffiths transversality).

(iii) $\exp \left(\sum_{1 \leq j \leq r} i y_{j} N_{j}\right) F \in D\left(\forall y_{j} \gg 0\right)$, (positivity). Here $\left(N_{j}\right)_{1 \leq j \leq r}$ is a system of generators of $\sigma$.

(1.4) Logarithmic structures.

Let $X$ be a ringed space over $\mathbf{C}$ with structure sheaf $\mathcal{O}_{X}$. We recall briefly the notion of logarithmic structures on $X$ of Fontaine-Illusie (for details, cf. [I], [K1]).

A pre-logarithmic structure on $X$ is a sheaf of commutative monoids $M_{X}$ together with a homomorphism $\alpha: M_{X} \rightarrow \mathcal{O}_{X}$ of monoids, where $\mathcal{O}_{X}$ is regarded as a sheaf of monoids by multiplication. A

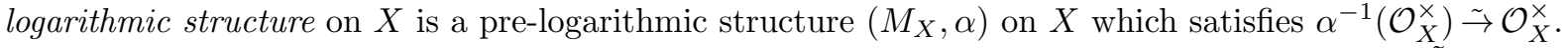

Let $\left(M_{X}, \alpha\right)$ be a pre-logarithmic structure on $X$. The associated logarithmic structure $\left(\tilde{M}_{X}, \tilde{\alpha}\right)$ is defined as the push-forward $\tilde{M}_{X}$ of $\mathcal{O}_{X}^{\times} \leftarrow \alpha^{-1}\left(\mathcal{O}_{X}^{\times}\right) \rightarrow M_{X}$, namely the diagram

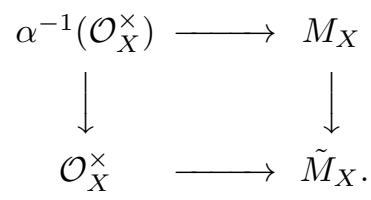

is co-cartesian, together with the homomorphism $\tilde{\alpha}: \tilde{M}_{X} \rightarrow \mathcal{O}_{X}$ induced by $\alpha: M_{X} \rightarrow \mathcal{O}_{X}$ and the inclusion $\mathcal{O}_{X}^{\times} \hookrightarrow \mathcal{O}_{X}$.

Let $f:\left(X, \mathcal{O}_{X}\right) \rightarrow\left(Y, \mathcal{O}_{Y}\right)$ be a morphism of ringed spaces and $(M, \alpha)$ be a logarithmic structure on $Y$. Then the sheaf-theoretic inverse image $f^{-1} M$ together with the composite morphism $f^{-1} M \rightarrow$ $f^{-1} \mathcal{O}_{Y} \rightarrow \mathcal{O}_{X}$ form a pre-logarithmic structure on $X$. The inverse image $f^{*}(M, \alpha)$ of $(M, \alpha)$ is defined as the logarithmic structure on $X$ associated to the above pre-logarithmic structure.

A monoid $\mathcal{S}$ is $f_{s}$ (= finitely-generated, integral and saturated) if it satisfies the following three conditions.

(i) $\mathcal{S}$ is finitely generated.

(ii) If $a, b, c \in \mathcal{S}$ and $a b=a c$, then $b=c$.

(iii) If $a \in \mathcal{S}^{\mathrm{gp}}$ and $a^{n} \in \mathcal{S}$ for some positive integer $n$, then $a \in \mathcal{S}$.

Here $\mathcal{S}^{\mathrm{gp}}$ is the group generated by $\mathcal{S}$. A logarithmic structure $\left(M_{X}, \alpha\right)$ on $X$ is $f_{s}$ if there exists an open covering $\left(U_{\lambda}\right)_{\lambda}$ of $X$ such that each restriction $\left.\left(M_{X}, \alpha\right)\right|_{U_{\lambda}}$ is the logarithmic structure associated to a pre-logarithmic structure of the form $\left(\mathcal{S}_{U_{\lambda}}, \alpha_{\lambda}\right)$ where $\mathcal{S}_{U_{\lambda}}$ is a constant sheaf of monoids on $U_{\lambda}$ induced by an fs monoid $\mathcal{S}$. In this case, $\left(\mathcal{S}_{U_{\lambda}}, \alpha_{\lambda}\right)$ is called a chart of $\left(M_{X}, \alpha\right)$ on $U_{\lambda}$.

(1.5) The ringed space $\left(X^{\log }, \mathcal{O}_{X}^{\log }\right)$.

Let $X$ be an fs logarithmic local ringed space (= a local ringed space endowed with an fs logarithmic structure) over $\mathbf{C}$ which satisfies the following two conditions:

(i) $\mathcal{O}_{X, x} / \mathfrak{m}_{x}=\mathbf{C}$.

(ii) For any open set $U \subset X$ and any $f \in \mathcal{O}_{X}(U)$, the evaluation map $U \rightarrow \mathbf{C}, x \mapsto f(x)$, is continuous.

We recall briefly the associated ringed space $\left(X^{\log }, \mathcal{O}_{X}^{\log }\right)$ introduced in $[\mathrm{KkNc}]$ (cf. also $[\mathrm{KyNy}]$ ).

As a set, $X^{\log }$ is the set of all pairs $(x, h)$, consisting of a point $x \in X$ and a homomorphism $h$ : $M_{X, x} \rightarrow \mathbf{S}^{1}$ whose restriction to $\mathcal{O}_{X}^{\times}$is $u \mapsto u(x) /|u(x)|$. Here $\mathbf{S}^{1}:=\{t \in \mathbf{C} ;|t|=1\}$.

The topology of $X^{\log }$ is defined as follows. We work locally on $X$. Take a chart $\mathcal{S} \rightarrow M_{X}$, then we have an injective map

$$
X^{\log } \hookrightarrow X \times \operatorname{Hom}\left(\mathcal{S}^{\mathrm{gp}}, \mathbf{S}^{1}\right),(x, h) \mapsto\left(x, h_{\mathcal{S}}\right),
$$

where $h_{\mathcal{S}}$ denotes the composite map $\mathcal{S}^{\mathrm{gp}} \rightarrow M_{X, x}^{\mathrm{gp}} \rightarrow \mathbf{S}^{1}$. We endow $X^{\log }$ with the topology as a subset of $X \times \operatorname{Hom}\left(\mathcal{S}^{\mathrm{gp}}, \mathbf{S}^{1}\right)$. It can be seen that this topology is independent of the choice of chart and hence is globally well-defined, and that the canonical map $\tau: X^{\log } \rightarrow X,(x, h) \mapsto x$ is surjective, continuous and proper. 
The sheaf of $\mathbf{C}$-algebras $\mathcal{O}_{X}^{\log }$ is defined as follows. Let $\mathcal{L}$ be the fiber product of

$$
\operatorname{Cont}(, i \mathbf{R}) \stackrel{\exp }{\longrightarrow} \operatorname{Cont}\left(, \mathbf{S}^{1}\right),
$$

where Cont $(, T)$, for a topological space $T$, denotes the sheaf on $X^{\log }$ of continuous maps to $T$, and $\tau^{-1}\left(M_{X}^{\mathrm{gp}}\right) \rightarrow \operatorname{Cont}\left(, \mathbf{S}^{1}\right)$ comes from the definition of $X^{\log }$. We define

$$
\mathcal{O}_{X}^{\log }:=\left(\tau^{-1}\left(\mathcal{O}_{X}\right) \otimes_{\mathbf{Z}} \operatorname{Sym}_{\mathbf{Z}}(\mathcal{L})\right) / \mathfrak{a},
$$

where $\operatorname{Sym}_{\mathbf{Z}}(\mathcal{L})$ denotes the symmetric algebra of $\mathcal{L}$ over $\mathbf{Z}$, and $\mathfrak{a}$ is the ideal of $\tau^{-1}\left(\mathcal{O}_{X}\right) \otimes_{\mathbf{Z}} \operatorname{Sym}_{\mathbf{Z}}(\mathcal{L})$ generated by the image of

$$
\tau^{-1}\left(\mathcal{O}_{X}\right) \rightarrow \tau^{-1}\left(\mathcal{O}_{X}\right) \otimes \mathbf{Z} \operatorname{Sym}_{\mathbf{Z}}(\mathcal{L}), \quad f \mapsto f \otimes 1-1 \otimes \iota(f) .
$$

Here the map $\iota: \tau^{-1}\left(\mathcal{O}_{X}\right) \rightarrow \mathcal{L}$ is the one induced by

$$
\begin{aligned}
& \tau^{-1}\left(\mathcal{O}_{X}\right) \rightarrow \operatorname{Cont}(, i \mathbf{R}), \quad f \mapsto \frac{1}{2}(f-\bar{f}), \quad \text { and } \\
& \tau^{-1}\left(\mathcal{O}_{X}\right) \stackrel{\exp }{\longrightarrow} \tau^{-1}\left(\mathcal{O}_{X}^{\times}\right) \subset \tau^{-1}\left(M_{X}^{\mathrm{gp}}\right) .
\end{aligned}
$$

Let $y \in X^{\log }$ and $x:=\tau(y) \in X$. There exist a family $\left(\ell_{j}\right)_{1 \leq j \leq n}$ of elements of $\mathcal{L}_{y}$, whose images in $\left(M_{X}^{\mathrm{gp}} / \mathcal{O}_{X}^{\times}\right)_{x}$ form a system of free generators, and which are algebraically independent over $\mathcal{O}_{X, x}$ and generate the stalk $\mathcal{O}_{X, y}^{\log }=\tau^{-1}\left(\mathcal{O}_{X, x}\right)\left[\ell_{1}, \ldots, \ell_{n}\right]$. Note that this is not a local ring if $\left(M_{X}^{\mathrm{gp}} / \mathcal{O}_{X}^{\times}\right)_{x} \neq\{1\}$. Example. Let $X:=\mathbf{C}$ be the complex plane and let $z$ be the coordinate of $X$. The fs logarithmic structure on $X$ associated to the divisor $\{0\}$ is defined by

$$
M_{X}:=\left\{f \in \mathcal{O}_{X} ; f \text { is invertible outside }\{0\}\right\} \hookrightarrow \mathcal{O}_{X}
$$

We can take a chart $\mathbf{N} \rightarrow M_{X}=\bigsqcup_{n>0} z^{n} \mathcal{O}_{X}^{\times}, n \mapsto z^{n}$. We have an isomorphism

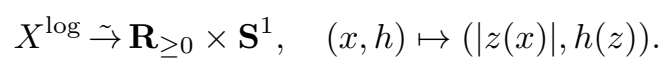

Let $\ell:=\log z$, then we have $\mathcal{O}_{X}^{\log }=\tau^{-1}\left(\mathcal{O}_{X}\right)[\ell]$.

\section{§2. Spaces of nilpotent orbits}

Let $\Sigma$ be a fan in $\mathfrak{g}_{\mathbf{Q}}$ and let $\Gamma$ be a subgroup of $G_{\mathbf{Z}}$ which is strongly compatible with $\Sigma$.

(2.1) The set $D_{\Sigma}$.

Definition. As a set, we define the space $D_{\Sigma}$ of nilpotent orbits in the directions in $\Sigma$ by

$$
D_{\Sigma}:=\{(\sigma, Z) ; \sigma \in \Sigma, Z \subset \check{D} \text { is a } \sigma \text {-nilpotent orbit }\} \text {. }
$$

Remark. Note that the set $D$ is contained in $D_{\Sigma}$ by $F \mapsto(\{0\},\{F\})$.

Let $\sigma$ be a nilpotent cone in $\mathfrak{g}_{\mathbf{Q}}$. Then, $\{$ faces of $\sigma\}$ is a fan, and we denote

$$
D_{\sigma}:=D_{\{\text {faces of } \sigma\}} \cdot
$$

(2.2) The toric variety toric $(\Gamma(\sigma))$. 
In order to fix notations, we review here toric varieties (cf. [AMRT]). For $\sigma \in \Sigma$, we see that $\Gamma(\sigma)$ is an fs monoid with $\Gamma(\sigma)^{\times}=\{1\}$. The associated toric variety is defined by

$$
\operatorname{toric}(\Gamma(\sigma)):=\operatorname{Spec}\left(\mathbf{C}\left[\Gamma(\sigma)^{\vee}\right]\right)_{\text {an }} .
$$

Here $\Gamma(\sigma)^{\vee}:=\operatorname{Hom}(\Gamma(\sigma), \mathbf{N})$. Note that the set of complex points of $\operatorname{toric}(\Gamma(\sigma))$ can be identified with $\operatorname{Hom}\left(\Gamma(\sigma)^{\vee}, \mathbf{C}^{\text {mult }}\right)$. Here $\mathbf{C}^{\text {mult }}$ means the set $\mathbf{C}$ of complex numbers with multiplication.

We have bijections

$$
\operatorname{toric}(\Gamma(\sigma)) \stackrel{\alpha}{\leftarrow} \bigsqcup_{\tau \prec \sigma}\left(\Gamma(\sigma)^{\mathrm{gp}} / \Gamma(\tau)^{\mathrm{gp}}\right) \otimes \mathbf{C}^{\times} \stackrel{\beta}{\rightarrow} \bigsqcup_{\tau \prec \sigma} \exp \left(\sigma_{\mathbf{C}}\right) /\left(\exp \left(\tau_{\mathbf{C}}\right) \Gamma(\sigma)^{\mathrm{gp}}\right)
$$

Here $\tau \prec \sigma$ means that $\tau$ is a face of $\sigma$, and $\alpha$ is defined as follows: For $a \in\left(\Gamma(\sigma)^{\mathrm{gp}} / \Gamma(\tau)^{\mathrm{gp}}\right) \otimes \mathbf{C}^{\times}$and $h \in \Gamma(\sigma)^{\vee}$,

$$
\alpha(\tau, a)(h):= \begin{cases}0 & \text { if } h(\Gamma(\tau)) \neq 0, \\ h(a) & \text { if } h(\Gamma(\tau))=0 .\end{cases}
$$

In the second case in the above, we regard $h$ as the induced homomorphism $h:\left(\Gamma(\sigma)^{\mathrm{gp}} / \Gamma(\tau)^{\mathrm{gp}}\right) \otimes \mathbf{C}^{\times} \rightarrow$ $\mathbf{Z} \otimes \mathbf{C}^{\times}=\mathbf{C}^{\times}$.

And $\beta$ is defined by

$$
\beta(\gamma \otimes \exp (2 \pi i z)):=\exp (z \log \gamma) \quad\left(\gamma \in \Gamma(\sigma)^{\mathrm{gp}}, z \in \mathbf{C}\right) .
$$

(2.3) The subset $E_{\sigma} \subset \operatorname{toric}(\Gamma(\sigma)) \times \check{D}$.

Definition. For $\sigma \in \Sigma$, we define the set $E_{\sigma}$ by

$$
E_{\sigma}:=\left\{(\theta, F) \in \operatorname{toric}(\Gamma(\sigma)) \times \check{D} ; \exp \left(\tau_{\mathbf{C}}\right) g F \text { is a } \tau \text {-nilpotent orbit }\right\}
$$

Here $\left(\tau, g \bmod \exp \left(\tau_{\mathbf{C}}\right) \Gamma(\sigma)^{\mathrm{gp}}\right)$ denotes the pair corresponding to $\theta$ under the composite map (2.2.1).

Define the map $E_{\sigma} \rightarrow \Gamma(\sigma)^{\mathrm{gP}} \backslash D_{\sigma}$ by $(\theta, F) \mapsto\left(\tau, \exp \left(\tau_{\mathbf{C}}\right) g F\right)$.

Define the action of $\sigma_{\mathrm{C}}$ on $E_{\sigma}$ over $\Gamma(\sigma)^{\mathrm{gp}} \backslash D_{\sigma}$ by

$$
a \cdot(\theta, F):=(\exp (2 \pi i a) \theta, \exp (-a) F) \quad\left(a \in \sigma_{\mathbf{C}}\right) .
$$

Here, for $b \in \sigma_{\mathbf{C}}$, $\exp (b) \theta$ is the element of $\operatorname{Hom}_{\mathbf{C}-a l g}\left(\mathbf{C}\left[\Gamma(\sigma)^{\vee}\right], \mathbf{C}\right)$ defined by

$$
(\exp (b) \theta)(h):=\exp (h(\exp (b))) \theta(h) \quad\left(h \in \Gamma(\sigma)^{\vee}\right) .
$$

Then, $E_{\sigma} \rightarrow \Gamma(\sigma)^{\mathrm{gp}} \backslash D_{\sigma}$ is surjective (more precisely, a $\sigma_{\mathbf{C}}$-torsor, see (6.1.1) below).

Example. In the case $\operatorname{rank}(\sigma)=1$, we have toric $(\Gamma(\sigma)) \simeq \mathbf{C}$. Let $\gamma$ be the generator of $\Gamma(\sigma)$ and let $N:=\log (\gamma)$. Then we have

$$
\begin{aligned}
& E_{\sigma}:=\{(\theta, F) \in \mathbf{C} \times \check{D} ; \text { If } \theta \neq 0, \exp ((\log (\theta) / 2 \pi i) N) F \in D . \\
&\text { If } \left.\theta=0, \exp \left(\sigma_{\mathbf{C}}\right) F \text { is a } \sigma \text {-nilpotent orbit. }\right\} .
\end{aligned}
$$

Here the cases $\theta \neq 0, \theta=0$ correspond $\tau=0, \tau=\sigma$, respectively. $\sigma_{\mathbf{C}} \simeq \mathbf{C} \ni a$ acts on $E_{\sigma}$ over $\Gamma(\sigma)^{\mathrm{gp}} \backslash D_{\sigma}$ by

$$
a \cdot(\theta, F)=(\exp (2 \pi i a) \theta, \exp (-a N) F)
$$




\section{§3. SPACES OF NILPOTENT $i$-ORBits}

Let $\Sigma$ be a fan in $\mathfrak{g}_{\mathbf{Q}}$ and let $\Gamma$ be a subgroup of $G_{\mathbf{Z}}$ which is strongly compatible with $\Sigma$ as before.

(3.1) The set $D_{\Sigma}^{\sharp}$.

Definition. As a set, we define the space $D_{\Sigma}^{\sharp}$ of nilpotent $i$-orbits in the directions in $\Sigma$ by

$$
D_{\Sigma}^{\sharp}:=\{(\sigma, Z) ; \sigma \in \Sigma, Z \subset \check{D} \text { is a } \sigma \text {-nilpotent } i \text {-orbit }\} .
$$

Define the map

$$
D_{\Sigma}^{\sharp} \rightarrow D_{\Sigma}, \quad(\sigma, Z) \mapsto\left(\sigma, \exp \left(\sigma_{\mathbf{C}}\right) Z\right) .
$$

Remark. Note that the set $D$ is contained in $D_{\Sigma}^{\sharp}$ by $F \mapsto(\{0\},\{F\})$.

For a nilpotent cone $\sigma$ in $\mathfrak{g}_{\mathbf{Q}}$, we denote

$$
D_{\sigma}^{\sharp}:=D_{\{\text {faces of } \sigma\}}^{\sharp} .
$$

(3.2) The real toric variety $\mid$ toric $\mid(\sigma)$.

In order to fix notations, we review here real toric varieties (for details, see [AMRT, I §1], [HZ, 2.1]). For $\sigma \in \Sigma$, we define

$$
|\operatorname{toric}|(\sigma):=\operatorname{Hom}\left(\sigma^{\vee},(\mathbf{R} \cup\{\infty\})^{\text {add }}\right) \supset \sigma_{\mathbf{R}}=\operatorname{Hom}\left(\sigma^{\vee}, \mathbf{R}^{\text {add }}\right) .
$$

Here $\sigma^{\vee}:=\operatorname{Hom}\left(\sigma, \mathbf{Q}_{\geq 0}^{\text {add }}\right)$. We have the bijection

$$
\mid \text { toric } \mid(\sigma) \stackrel{\alpha}{\leftarrow} \bigsqcup_{\tau \prec \sigma} \sigma_{\mathbf{R}} / \tau_{\mathbf{R}},
$$

defined by

By using the isomorphism

$$
\alpha\left(\tau, H \bmod \tau_{\mathbf{R}}\right)(h):=\left\{\begin{array}{ll}
h(H) & \text { if } h(\tau)=0, \\
\infty & \text { if } h(\tau) \neq 0,
\end{array} \quad\left(h \in \sigma^{\vee}\right) .\right.
$$

$$
(\mathbf{R} \cup\{\infty\})^{\text {add }} \stackrel{\sim}{\rightarrow} \mathbf{R}_{\geq 0}^{\text {mult }}, \quad y \mapsto e^{-2 \pi y},
$$

we have

$$
\mid \text { toric } \mid(\sigma) \simeq \operatorname{Hom}\left(\sigma^{\vee}, \mathbf{R}_{\geq 0}^{\text {mult }}\right) \simeq \operatorname{Hom}\left(\Gamma(\sigma)^{\vee}, \mathbf{R}_{\geq 0}^{\text {mult }}\right) .
$$

If we identify toric $(\Gamma(\sigma))$ with $\operatorname{Hom}\left(\Gamma(\sigma)^{\vee}, \mathbf{C}^{\text {mult }}\right)$, we see that the maps

$$
\mathbf{C}^{\text {mult }} \rightarrow \mathbf{R}_{\geq 0}^{\text {mult }}, \quad q \mapsto|q|, \quad \text { and } \quad \mathbf{R}_{\geq 0}^{\text {mult }} \rightarrow \mathbf{C}^{\text {mult }}, \quad r \mapsto r,
$$

induce the maps

$$
\operatorname{toric}(\Gamma(\sigma)) \rightleftarrows|\operatorname{toric}|(\sigma) \text {. }
$$

(3.3) The subset $E_{\Sigma}^{\sharp} \subset \mid$ toric $\mid(\sigma) \times \check{D}$.

Definition. For $\sigma \in \Sigma$, we define the set $E_{\sigma}^{\sharp}$ by

$$
E_{\sigma}^{\sharp}:=\left\{(b, F) \in \mid \text { toric } \mid(\sigma) \times \check{D} ; \exp \left(i \tau_{\mathbf{R}}\right) \exp (i H) F \text { is a } \tau \text {-nilpotent } \text { - -orbit }\right\} .
$$

Here $\left(\tau, H \bmod \tau_{\mathbf{R}}\right)$ is the point in $\bigsqcup_{\tau \prec \sigma} \sigma_{\mathbf{R}} / \tau_{\mathbf{R}}$ corresponding to $b$ in $\mid$ toric $\mid(\sigma)$ under the bijection (3.2.1). Define the map $E_{\sigma}^{\sharp} \rightarrow D_{\sigma}^{\sharp}$ by $(b, F) \mapsto \exp \left(i \tau_{\mathbf{R}}\right) \exp (i H) F$.

Define the action of $\sigma_{\mathbf{R}}$ on $E_{\sigma}^{\sharp}$ over $D_{\sigma}^{\sharp}$ by

$$
a \cdot\left(\left(\tau, H \bmod \tau_{\mathbf{R}}\right), F\right):=\left(\left(\tau,(H+a) \bmod \tau_{\mathbf{R}}\right), \exp (-i a) F\right) \quad\left(a \in \sigma_{\mathbf{R}}\right) .
$$

Then, $E_{\sigma}^{\sharp} \rightarrow D_{\sigma}^{\sharp}$ is surjective (more precisely, a $\sigma_{\mathbf{R}}$-torsor, see (6.1.1) below). The maps in (3.2.2) induce the maps

$$
E_{\sigma} \rightleftarrows E_{\sigma}^{\sharp},
$$

whose composite $E_{\sigma}^{\sharp} \rightarrow E_{\sigma} \rightarrow E_{\sigma}^{\sharp}$ is the identity. 


\section{$\S 4$. Topology, Generalized fs logarithmic analytic spaces}

(4.1) The strong topology.

Definition. Let $X$ be an analytic space and $S$ be a subset on $X$. The strong topology on $S$ is the strongest topology on $S$ for which the map $\lambda: Y \rightarrow S$ is continuous for any analytic space $Y$ and for any analytic morphism $\lambda: Y \rightarrow X$ with $\lambda(Y) \subset S$.

Remark. In the notation of the above definition, if $S$ is an analytic subspace of $X$ then the strong topology on $S$ coincides with the usual topology, i.e., the topology as a subspace of $X$.

Example. Let $X:=\mathbf{C}^{2}$ be the complex two-dimensional plane and let $S:=\mathbf{C}^{2}-\{0\} \times \mathbf{C}+\{(0,0)\} \subset X$. For the strong topology on $S$ and the usual topology on $S$ as a subspace of $X$, we can observe the following:

If $k \in \mathbf{Z}_{\geq 1}$ and $z \in \mathbf{C}$ converges to 0 then $\left(z^{k}, z\right)$ converges to $(0,0)$ in the strong topology on $S$.

If $a \in \mathbf{R}_{>1}$ and $s \in \mathbf{R}, s \rightarrow \infty$, then $\left(1 / a^{s}, 1 / s\right)$ does not converge to $(0,0)$ in the strong topology on $S$, whereas $\left(1 / a^{s}, 1 / s\right)$ converges to $(0,0)$ in the usual topology on $S$ as a subspace of $X$.

(4.2) Generalized (fs logarithmic) analytic spaces.

Definitions. (4.2.1) Let $\mathcal{C}$ be a category and let $\mathcal{A}$ be a full subcategory of $\mathcal{C}$. For an object $S$ of $\mathcal{C}$, let $h_{\mathcal{A}}^{S}$ be the contravariant functor

$$
\mathcal{A} \rightarrow \text { Sets }, \quad Y \mapsto h_{\mathcal{A}}^{S}(Y):=\operatorname{Mor}(Y, S)
$$

Denote by $\overline{\mathcal{A}} \supset \mathcal{A}$ the full subcategory of $\mathcal{C}$ consisting of all objects $S$ satisfying

$$
\operatorname{Mor}(S, Z) \stackrel{\sim}{\rightarrow} \operatorname{Mor}\left(h_{\mathcal{A}}^{S}, h_{\mathcal{A}}^{Z}\right) \quad \text { for any object } Z \text { of } \mathcal{C} .
$$

$$
\mathcal{C}:=(\text { local ringed spaces over } \mathbf{C}) \supset \mathcal{A}:=\text { (analytic spaces), }
$$

we call an object of $\overline{\mathcal{A}}$ a generalized analytic space.

(4.2.3) For

$$
\begin{aligned}
\mathcal{C}:= & (\text { fs logarithmic local ringed spaces over } \mathbf{C}) \\
& \supset \mathcal{A}:=(\text { fs logarithmic analytic spaces })
\end{aligned}
$$

we call an object of $\overline{\mathcal{A}}$ a generalized fs logarithmic analytic space.

It can be proved that, if we forget logarithmic structures, generalized fs logarithmic analytic spaces become generalized analytic spaces. Generalized analytic spaces satisfy the conditions (1.5) (i), (ii).

Remark. In the example of (4.1), $S$, endowed with the strong topology and the pull-back sheaf of rings $\mathcal{O}$, is a generalized analytic space. $S$, endowed moreover with the pull-back fs logarithmic structure $M$ from those on $X$, is a generalized fs logarithmic analytic space. Here the fs logarithmic structure on $X$ is the one associated to the divisor $\{0\} \times \mathbf{C} \subset X$.

\section{§5. Polarized logarithmic Hodge structures}

(5.1) Polarized logarithmic Hodge structures.

Definition. (5.1.1) Let $X$ be a generalized $f s$ logarithmic analytic space. A pre-polarized logarithmic Hodge structure (pre-PLH for short) on $X$, of weight $w$ and of Hodge type $\left(h^{p, q}\right)_{p+q=w}$ is a triple $\left(H_{\mathbf{Z}},\langle\rangle, F,\right)$ consisting of a locally constant sheaf of free $\mathbf{Z}$-modules $H_{\mathbf{Z}}$ on $X^{\log }$, of a non-degenerate bilinear form $\langle\rangle:, H_{\mathbf{Q}} \times H_{\mathbf{Q}} \rightarrow \mathbf{Q}$, symmetric for even $w$ and anti-symmetric for odd $w$ and of a decreasing filtration $F$ of the $\mathcal{O}_{X}^{\log }$-module $\mathcal{O}_{X}^{\log } \otimes H_{\mathbf{Z}}$, which satisfy the following three conditions.

(i) Each $F^{p}$ is the pull-back under $\tau:\left(X^{\log }, \mathcal{O}_{X}^{\log }\right) \rightarrow\left(X, \mathcal{O}_{X}\right)$ of a locally free $\mathcal{O}_{X}$-module of rank $\sum_{r \geq p} h^{r, w-r}$. 
(ii) Each $F^{p}$ is, locally on $X^{\log }$, an $\mathcal{O}_{X}^{\log }$-direct summand of $\mathcal{O}_{X}^{\log } \otimes H_{\mathbf{Z}}$.

(iii) $\left\langle F^{p}, F^{q}\right\rangle=0$ if $p+q>w$.

Let $X$ be an fs logarithmic analytic space. Let $\Omega_{X}^{1}$ be the sheaf of Kähler differentials on $X$. The sheaf of logarithmic differential forms on $X$ is defined by

$$
\omega_{X}^{1}:=\left(\Omega_{X}^{1} \oplus M_{X}^{\mathrm{gp}}\right) / N
$$

where $N$ is the $\mathcal{O}_{X}$-submodule generated by $\left\{(-d \alpha(x), \alpha(x) \otimes x) ; x \in M_{X}\right\}$. The derivation $d: \mathcal{O}_{X} \rightarrow \Omega_{X}^{1}$ induces the one $d: \mathcal{O}_{X} \rightarrow \omega_{X}^{1}$ via the map $\Omega_{X} \rightarrow \omega_{X}^{1}$. The sheaf $\omega_{X}^{1, \log }$ on $X^{\log }$ is defined by

$$
\omega_{X}^{1, \log }:=\tau^{*}\left(\omega_{X}^{1}\right)=\mathcal{O}_{X}^{\log } \otimes_{\tau^{-1}\left(\mathcal{O}_{X}\right)} \tau^{-1}\left(\omega_{X}^{1}\right)
$$

The derivation

$$
d: \mathcal{O}_{X}^{\log } \rightarrow \omega_{X}^{1, \log }
$$

is induced by $d: \mathcal{O}_{X} \rightarrow \omega_{X}^{1}$ and by $\mathcal{L} \stackrel{\exp }{\longrightarrow} \tau^{-1}\left(M_{X}^{\text {gp }}\right) \stackrel{d \log }{\longrightarrow} \tau^{-1}\left(\omega_{X}^{1}\right)$, where $d \log : x \mapsto(0,1 \otimes x)$.

Let now $x$ be an fs logarithmic analytic space whose underlying analytic space is $\operatorname{Spec}(\mathbf{C})$ and let $r:=\operatorname{rank}_{\mathbf{Z}}\left(M_{x}^{\mathrm{gp}} / \mathcal{O}_{x}^{\times}\right)$. Then $x^{\log }=\left(\mathbf{S}^{1}\right)^{r}$. Let $y \in x^{\log }$. We define

$$
\begin{aligned}
& \operatorname{sp}(y):=\operatorname{Hom}_{\mathbf{C}-\operatorname{alg}}\left(\mathcal{O}_{x, y}^{\log }, \mathbf{C}\right), \\
& \operatorname{sp}(x):=\left\{t \in \operatorname{Hom}\left(M_{x}, \mathbf{C}^{\text {mult }}\right) ;\left.t\right|_{\mathbf{C}^{\times}}=i d\right\}, \quad \text { and } \\
& \operatorname{sp}(y) \rightarrow \operatorname{sp}(x), \quad s \mapsto t:=\left(M_{x} \ni a \mapsto \exp (s(\log (a))) \in \mathbf{C}^{\times} \subset \mathbf{C}\right) .
\end{aligned}
$$

Note that the structure homomorphism $\alpha: M_{x} \rightarrow \mathbf{C}$ of the logarithmic structure of $x$ is an element of $\operatorname{sp}(x)$, which does not belong to the image of the canonical map $\operatorname{sp}(y) \rightarrow \operatorname{sp}(x)$ if $r>0$. Note also that $\operatorname{sp}(x) \simeq \mathbf{C}^{r}$, whereas $\operatorname{sp}(y)\left(y \in x^{\log }\right)$ form a $\mathbf{C} \otimes_{\mathbf{z}} \pi_{1}\left(x^{\log }\right)$-torsor over $x^{\log }$ (cf. (5.2) below).

For a pre-PLH $\left(H_{\mathbf{z}},\langle\rangle, F,\right)$ on $x$, for $y \in x^{\log }$ and for $s \in \operatorname{sp}(y)$, we have a decreasing filtration

$$
F(s):=\left(\mathbf{C} \otimes_{\mathcal{O}_{x, y}^{\log }} F_{y}^{p}\right)_{p \in \mathbf{Z}}, \quad \text { where } \quad s: \mathcal{O}_{x, y}^{\log } \rightarrow \mathbf{C},
$$

on the $\mathbf{C}$-vector space $H_{\mathbf{C}, y}:=\mathbf{C} \otimes_{\mathbf{Z}} H_{\mathbf{Z}, y}$.

Definition. (5.1.2) Let $x$ be an $f$ s logarithmic analytic space whose underlying analytic space is $\operatorname{Spec}(\mathbf{C})$. A pre-PLH $\left(H_{\mathbf{Z}},\langle\rangle, F,\right)$ on $x$ is called a polarized logarithmic Hodge structure (PLH for short) on $x$ if it satisfies the following two conditions.

(i) $\quad(d \otimes 1)\left(F^{p}\right) \subset \omega_{x}^{1, \log } \otimes_{\mathcal{O}_{x}^{\log }} F^{p-1}$ for all $p$

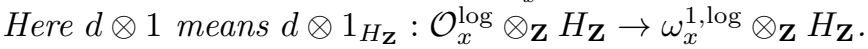

(ii) For $y \in x^{\log }$ and for $s \in \operatorname{sp}(y)$ whose image in $\operatorname{sp}(x)$ is sufficiently near to the structure homomorphism $\alpha \in \operatorname{sp}(x),\left(H_{\mathbf{Z}, y},\langle,\rangle_{y}, F(s)\right)$ is a polarized Hodge structure in the usual sense.

Note that the validity of the condition (5.1.2) (ii) is independent of the choice of $y$.

Definition. (5.1.3) Let $X$ be a generalized $f$ s logarithmic analytic space. A pre-PLH $\left(H_{\mathbf{Z}},\langle\rangle, F,\right)$ on $X$ is called a polarized logarithmic Hodge structure (PLH for short) on $X$ if, for any $x \in X$, the inverse image of $\left(H_{\mathbf{Z}},\langle\rangle, F,\right)$ on $x$ is a PLH. Here we regard $x$ as an $f_{s}$ logarithmic analytic space with ring $\mathbf{C}$ and with the inverse image of the logarithmic structure of $X$.

(5.2) Polarized logarithmic Hodge structures of type $\Phi$.

Let $x$ be an fs logarithmic analytic space whose underlying analytic space is $\operatorname{Spec}(\mathbf{C})$. Then $x^{\log } \simeq$ $\left(\mathbf{S}^{1}\right)^{r}$, where $r=\operatorname{rank}\left(M_{x}^{\mathrm{gp}} / \mathcal{O}_{x}^{\times}\right)$, as before. Let $y \in x^{\log }$. We define an action of $\mathbf{C} \otimes \pi_{1}\left(x^{\log }\right)$ on $\operatorname{sp}(y)$ as follows. Let $\mathcal{L}$ be the sheaf constructed in (1.5). The exact sequence

$$
0 \rightarrow \mathbf{Z} \stackrel{2 \pi i}{\longrightarrow} \mathcal{L} \stackrel{\exp }{\longrightarrow} \tau^{-1}\left(M_{x}^{\mathrm{gp}}\right) \rightarrow 1
$$


on $x^{\log }$ induces an exact sequence of cohomology groups

$$
\mathcal{O}_{x} \stackrel{\exp }{\rightarrow} M_{x}^{\text {gp }} \rightarrow H^{1}\left(x^{\log }, \mathbf{Z}\right) \simeq \operatorname{Hom}\left(\pi_{1}\left(x^{\log }\right), \mathbf{Z}\right) .
$$

Hence we have

$$
\pi_{1}\left(x^{\log }\right) \simeq \operatorname{Hom}\left(M_{x}^{\mathrm{gp}} / \mathcal{O}_{x}^{\times}, \mathbf{Z}\right) \tilde{\leftarrow} \operatorname{Hom}\left(\mathcal{L}_{y} / \mathcal{O}_{x}^{\text {add }}, \mathbf{Z}\right),
$$

where the last isomorphism is induced by exp. On the other hand, the restriction of $s \in \operatorname{sp}(y)$ to $\mathcal{L}_{y}$ yields a bijection

$$
\operatorname{sp}(y) \stackrel{\sim}{\rightarrow} \text { (splittings of the group homomorphism } \iota: \mathbf{C}^{\text {add }}=\mathcal{O}_{x}^{\text {add }} \rightarrow \mathcal{L}_{y} \text { ). }
$$

Now we define the action of $\varphi \in \mathbf{C} \otimes \mathbf{z} \pi_{1}\left(x^{\log }\right) \stackrel{\nu}{\rightarrow} \operatorname{Hom}\left(\mathcal{L}_{y} / \mathcal{O}_{x}^{\text {add }}, \mathbf{C}^{\text {add }}\right)$ on $s \in \operatorname{sp}(y)$ by

$$
\left.(\varphi s)\right|_{\mathcal{L}_{y}}:=(\varphi-\mathrm{id})+\left.s\right|_{\mathcal{L}_{y}}
$$

Note that $(\varphi-\mathrm{id}): \mathcal{L}_{y} \rightarrow \mathbf{C}^{\text {add }}$ is the lifting of $\nu(\varphi)$. Then, the $\operatorname{sp}(y)\left(y \in x^{\log }\right)$ form a $\mathbf{C} \otimes_{\mathbf{Z}} \pi_{1}\left(x^{\log }\right)$-torsor over $x^{\log } \simeq\left(\mathbf{S}^{1}\right)^{r}$ under this action.

The following proposition gives a connection between a PLH and nilpotent orbits.

Proposition. For $x \in X, y \in x^{\log }, s \in \operatorname{sp}(y)$ and $\varphi=\sum_{1 \leq j \leq n} z_{j} \otimes \gamma_{j} \in \mathbf{C} \otimes \pi_{1}\left(x^{\log }\right)$, we have

$$
F(\varphi s)=\exp \left(-\sum_{1 \leq j \leq n} z_{j} \log \left(\gamma_{j}\right)\right) F(s) \text {. }
$$

Let

$$
\Phi:=\left(w,\left(h^{p, q}\right)_{p+q=w}, H_{0},\langle,\rangle_{0}, \Gamma, \Sigma\right)
$$

be a 6-tuple consisting of a weight, a set of Hodge numbers, a free $\mathbf{Z}$-module $H_{0}$ of rank $\sum h^{p, q}$, a Q-bilinear form $\langle,\rangle_{0}$ on $H_{0, \mathbf{Q}}$ as in (1.1), a subgroup of $G_{\mathbf{Z}}$ and a fan with which the subgroup $\Gamma$ is strongly compatible.

Definition. Let $X$ be a generalized $f$ s logarithmic analytic space. A PLH of type $\Phi$ on $X$ is a 4-tuple $\left(H_{\mathbf{Z}},\langle\rangle, F,, \mu\right)$ consisting of a pre-PLH $\left(H_{\mathbf{Z}},\langle\rangle, F,\right)$ on $X$ of weight $w$ and of Hodge type $\left(h^{p, q}\right)_{p, q}$, and of a global section $\mu$ of the sheaf

$$
\Gamma \backslash \underline{\operatorname{Isom}}\left(\left(H_{\mathbf{Z}},\langle,\rangle\right),\left(H_{0},\langle,\rangle_{0}\right)\right)
$$

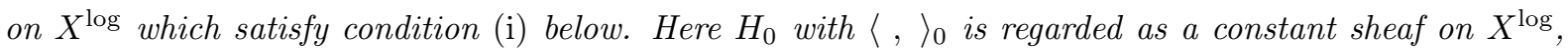
$\underline{\text { Isom }}$ is the sheaf of isomorphisms, and $\gamma \in \Gamma$ acts on $\underline{\operatorname{Isom}}(\cdots)$ by $h \mapsto \gamma \circ h$.

(i) For any $x \in X$ and any $y \in x^{\log }=\tau^{-1}(x)$, if

$$
\tilde{\mu}_{y}:\left(H_{\mathbf{Z}, y},\langle,\rangle_{y}\right) \stackrel{\sim}{\rightarrow}\left(H_{0},\langle,\rangle_{0}\right)
$$

denotes a representative of the stalk of $\mu$ at $y$, then there exists $\sigma \in \Sigma$ such that the image of the composite map

$$
\operatorname{Hom}\left(M_{X, x} / \mathcal{O}_{X, x}^{\times}, \mathbf{Z}_{\leq 0}\right) \hookrightarrow \pi_{1}\left(x^{\log }\right) \rightarrow \operatorname{Aut}_{\mathbf{Z}}\left(H_{\mathbf{Z}, y},\langle,\rangle_{y}\right) \stackrel{\text { by } \tilde{\mu}_{y}}{\simeq} \operatorname{Aut}_{\mathbf{Z}}\left(H_{0},\langle,\rangle_{0}\right)
$$

is contained in $\Gamma(\sigma)$ and that the filtrations $\tilde{\mu}_{y}\left(\left.F\right|_{x^{\log }}(s)\right)(s \in \operatorname{sp}(y))$ on $H_{0, \mathbf{C}}$ form a $\sigma$-nilpotent orbit (cf. Definition in (1.3)).

Remark. Using the above Proposition, we can prove that the conditions (i), (ii) in Definition (5.1.2) follow from the conditions (ii) (Griffiths transversality), (iii) (positivity) in Definition (1.3), respectively. Hence if $\left(H_{\mathbf{Z}},\langle\rangle, F,, \mu\right)$ is a PLH on $X$ of type $\Phi$ in the above definition, then $\left(H_{\mathbf{Z}},\langle\rangle, F,\right)$ is actually a PLH on $X$ in the sense of (5.1.2). 


\section{§6. MAin Result}

(6.1) Let $\Phi:=\left(w,\left(h^{p, q}\right)_{p+q=w}, H_{0},\langle,\rangle_{0}, \Gamma, \Sigma\right)$ be as in (5.2.1) and let $\sigma \in \Sigma$.

We endow $E_{\sigma} \subset \check{E}_{\sigma}:=\operatorname{toric}(\Gamma(\sigma)) \times \check{D}$ with the strong topology, the pull-back sheaf of rings $\mathcal{O}$ and the pull-back fs logarithmic structure $M$ from those on $\check{E}_{\sigma}$. Here the fs logarithmic structure on $\check{E}_{\sigma}$ is the pull-back of the one on toric $(\Gamma(\sigma))$ associated to the normal crossing divisor toric $(\Gamma(\sigma))-\Gamma(\sigma)^{\mathrm{gp}} \otimes \mathbf{C}^{\times}$.

Via $\pi_{\sigma}: E_{\sigma} \rightarrow \Gamma(\sigma)^{\mathrm{gp}} \backslash D_{\sigma} \rightarrow \Gamma \backslash D_{\Sigma}$, we endow $\Gamma(\sigma)^{\mathrm{gp}} \backslash D_{\sigma}$ with the quotient topology, and $\Gamma \backslash D_{\Sigma}$ with the strongest topology for which $\pi_{\sigma}$ is continuous for all $\sigma \in \Sigma$. For any open set $U$ of $\Gamma \backslash D_{\Sigma}$, let $U_{\sigma}:=\pi_{\sigma}^{-1}(U)$ and define

$$
\mathcal{O}(U)(\text { resp. } M(U)):=\left\{\operatorname{map} f: U \rightarrow \mathbf{C} ; f \circ \pi_{\sigma} \in \mathcal{O}\left(U_{\sigma}\right)\left(\text { resp. } \in M\left(U_{\sigma}\right)\right)(\forall \sigma \in \Sigma)\right\} .
$$

Here we regard $M\left(U_{\sigma}\right)$ as a subset of $\mathcal{O}\left(U_{\sigma}\right)$ via $\alpha: M\left(U_{\sigma}\right) \rightarrow \mathcal{O}\left(U_{\sigma}\right)$ which is injective.

We introduce the quotient topology on $E_{\sigma}^{\sharp}$ via the surjection $E_{\sigma} \rightarrow E_{\sigma}^{\sharp}$ in (3.3.1). Via $\pi_{\sigma}^{\sharp}: E_{\sigma}^{\sharp} \rightarrow$ $D_{\sigma}^{\sharp} \rightarrow \Gamma \backslash D_{\Sigma}^{\sharp}$, we endow $D_{\sigma}^{\sharp}$ with the quotient topology, and $D_{\Sigma}^{\sharp}$ with the strongest topology for which $\pi_{\sigma}^{\sharp}$ is continuous for all $\sigma \in \Sigma$.

A subgroup $\Gamma$ of $G_{\mathbf{Z}}$ is called neat if the subgroup of $\mathbf{C}^{\times}$, generated by all the eigenvalues of all $\gamma \in \Gamma$, is torsion free. It is known that there exist neat subgroups of $G_{\mathbf{Z}}$ with finite index.

Proposition. Assume $\Gamma$ is neat. Then, in the above notation, we have

(6.1.1) $E_{\sigma} \rightarrow \Gamma(\sigma)^{\mathrm{gp}} \backslash D_{\sigma}$ (resp. $\left.E_{\sigma}^{\sharp} \rightarrow D_{\sigma}^{\sharp}\right)$ is a $\sigma_{\mathbf{C}}$-torsor (resp. $\sigma_{\mathbf{R}}$-torsor) in the category of topological spaces.

(6.1.2) The maps $\Gamma(\sigma)^{\mathrm{gp}} \backslash D_{\sigma} \rightarrow \Gamma \backslash D_{\Sigma}$ and $D_{\sigma}^{\sharp} \rightarrow \Gamma \backslash D_{\Sigma}^{\sharp}$ are open.

(6.2) Our main result is

Theorem. Let $\Phi:=\left(w,\left(h^{p, q}\right)_{p+q=w}, H_{0},\langle,\rangle_{0}, \Gamma, \Sigma\right)$ be as in (5.2.1). We assume $\Gamma$ is neat. Then we have

(6.2.1) $\Gamma \backslash D_{\Sigma}$ is a generalized fs logarithmic analytic space, which is Hausdorff, but not locally compact in general. There is a homeomorphism of the topological spaces $\left(\Gamma \backslash D_{\Sigma}\right)^{\log }$ and $\Gamma \backslash D_{\Sigma}^{\sharp}$, which is compatible with the canonical map $\tau:\left(\Gamma \backslash D_{\Sigma}\right)^{\log } \rightarrow \Gamma \backslash D_{\Sigma}$ and the map $\Gamma \backslash D_{\Sigma}^{\sharp} \rightarrow \Gamma \backslash D_{\Sigma}$ induced by $D_{\Sigma}^{\sharp} \rightarrow D_{\Sigma}$ in (3.1).

(6.2.2) Let $\overline{\mathcal{A}}:=$ (generalized fs logarithmic analytic spaces) and define the contravariant functor $\underline{\mathrm{PLH}}_{\Phi}$ : $\overline{\mathcal{A}} \rightarrow$ Sets by

$$
\underline{\mathrm{PLH}}_{\Phi}(X):=(\text { isomorphism classes of PLH on X of type } \Phi) \text {. }
$$

Then this functor $\underline{\mathrm{PLH}}_{\Phi}$ is represented by $\Gamma \backslash D_{\Sigma}$.

Remark. In the case where $D$ is a Hermitian symmetric domain, the strong topology on $E_{\sigma} \subset \check{E}_{\sigma}$ coincides with the usual topology on $E_{\sigma}$ as a subspace of $\check{E}_{\sigma}$, and hence they coincide on $\Gamma \backslash D_{\Sigma}$. Thus our space $\Gamma \backslash D_{\Sigma}$ becomes an fs logarithmic analytic space whose underlying analytic space is nothing but a toroidal compactification of Mumford et al. for a 'big fan' $\Sigma$. Even in this case, our construction has the advantage that it carries the universal family of PLH.

\section{$\S 7$. EXAMPLES}

(7.1) Example of weight 3.

In the case of Hodge structures of wight 2, Cattani and Kaplan constructed a partial compactification $\Gamma \backslash D_{C K}(\Gamma \backslash \bar{D}$ in their notation in [CK]) as a topological space, which is a generalization of the Satake type. For the example of weight 3 below, their argument is applicable to obtain $\Gamma \backslash D_{C K}$. Consider the case of weight 2, or weight 3 of the type below. Let $\Xi$ be the fan in Example in (1.2) and let $\Gamma$ be a subgroup of $G_{\mathbf{Z}}$ with finite index. Then, there is the standard map $\Gamma \backslash D_{\Xi} \rightarrow \Gamma \backslash D_{\mathrm{CK}}$ from our space to the Cattani-Kaplan, which is continuous.

In the following, we give an example which shows that the strong topology is needed. Let $w=3$ and $h^{p, q}=1(p+q=3, p, q \geq 0), h^{p, q}=0$ otherwise. Let $e_{1}, e_{2}, f_{1}, f_{2}$ be a free $\mathbf{Z}$-basis of $H_{0}$ and let $\langle,\rangle_{0}$ be an anti-symmetric bilinear form on $H_{0}$ defined by

$$
\left\langle e_{1}, e_{2}\right\rangle_{0}=\left\langle f_{1}, f_{2}\right\rangle_{0}=1, \quad\left\langle e_{j}, e_{k}\right\rangle_{0}=0 \quad(\forall j, \forall k) .
$$


Let $N$ be a nilpotent endomorphism of $H_{0}$ defined by

$$
N f_{1}=f_{2}, \quad N f_{2}=N e_{j}=0 \quad(\forall j) .
$$

For $s \in \mathbf{C}$, we define a family of Hodge filtrations $F_{s} \in \check{D}$ by

$$
\begin{aligned}
F_{s}^{3} & :=\left\{s f_{1}+e_{1}-i e_{2}\right\}_{\mathbf{C}} \subset F_{s}^{2}:=\left\{f_{1}, e_{1}-i e_{2}\right\}_{\mathbf{C}} \\
& \subset F_{s}^{1}:=\left\{f_{1}, e_{1}-i e_{2}, s e_{2}-f_{2}\right\}_{\mathbf{C}} \subset F_{s}^{0}:=H_{0, \mathbf{C}}
\end{aligned}
$$

where $\{\ldots\}_{\mathbf{C}}$ means the $\mathbf{C}$-linear span. Using this family, we can prove

Proposition. (7.1.1) The following are equivalent:

(a) $\left(N, F_{s}\right)$ generates a nilpotent orbit.

(b) $\left(N, F_{s}\right)$ satisfies Griffiths transversality.

(c) $s=0$.

(7.1.2) We use the notation in (7.1.1). For $y \in \mathbf{R}$, we see $e^{i y N} F_{s} \in D$ if and only if $0<y<|s|^{-2}$. (Here we understand $|s|^{-2}=\infty$ for $\left.s=0\right)$.

(7.1.3) Let $\Xi$ be the fan in Example in (1.2). If we endow $E_{\sigma}(\sigma \in \Xi)$ with the usual topology as a subset of $\check{E}_{\sigma}$ and endow $\Gamma \backslash D_{\Xi}$ with the quotient topology via $\bigsqcup_{\sigma \in \Xi} E_{\sigma} \rightarrow \Gamma \backslash D_{\Xi}$, then $\Gamma \backslash D_{\Xi}$ is not Hausdorff and the standard map $\Gamma \backslash D_{\Xi} \rightarrow \Gamma \backslash D_{\mathrm{CK}}$ is not continuous.

The example in this subsection is a degeneration of the Hodge structures of weight 3 of the mirrors of quintic hypersurfaces in $\mathbf{P}^{4}$ (cf., for example, $[\mathrm{M}]$ ).

(7.2) Example of weight 2.

Let $\Xi$ be the fan in Example in (1.2). In this subsection, we give an example of $\Gamma \backslash D \Xi$ which is locally isomorphic to $\mathbf{C} \times\left(\mathbf{C}^{2}-\{0\} \times \mathbf{C}+\{(0,0)\}\right)$ (cf. Example in (4.1)).

Put

$$
\begin{aligned}
& S:=\mathbf{C} \times \mathbf{C}-\{0\} \times \mathbf{C}+\{(0,0)\}, \\
& \tilde{S}:=\mathbf{R}_{\geq 0} \times \mathbf{C}-\{0\} \times \mathbf{C}+\{(0,0)\} .
\end{aligned}
$$

We define a topology $\mathcal{T}$ (resp. $\tilde{\mathcal{T}}$ ) on $S$ (resp. on $\tilde{S}$ ) as follow. On $S-\{(0,0)\}$ (resp. on $\tilde{S}-\{(0,0)\}$ ), $\mathcal{T}$ (resp. $\tilde{\mathcal{T}}$ ) coincides with the usual topology, and, at the point $(0,0)$, a fundamental system of neighborhoods is given by

$$
\begin{aligned}
W(\varepsilon) & :=\bigcup_{n \geq 1}\left\{(v, z) ;|v|<\varepsilon_{n},|z|<\varepsilon_{n},|z|^{n}<|v|\right\} . \\
(\operatorname{resp} . \tilde{W}(\varepsilon) & \left.:=\bigcup_{n \geq 1}\left\{(r, z) ; r<\varepsilon_{n},|z|<\varepsilon_{n},|z|^{n}<r\right\} .\right)
\end{aligned}
$$

Here $\varepsilon=\left(\varepsilon_{n}\right)_{n \geq 1}$ runs over all families of positive real numbers. Then, $\mathcal{T}$ coincides with the strong topology of $S \subset \mathbf{C}^{2}$ considered in Example in (4.1).

Let $w=2, h^{2,0}=h^{0,2}=2, h^{1,1}=1$ and $h^{p, q}=0$ otherwise. Let $e_{j}(1 \leq j \leq 5)$ be a free $\mathbf{Z}$-basis of $H_{0}$ and let $\langle,\rangle_{0}$ be a symmetric bilinear form on $H_{0}$ defined by

$$
\left\langle e_{j}, e_{j}\right\rangle_{0}=1 \quad(1 \leq j \leq 3), \quad\left\langle e_{4}, e_{5}\right\rangle_{0}=\left\langle e_{5}, e_{4}\right\rangle_{0}=1,\left\langle e_{j}, e_{k}\right\rangle_{0}=0 \text { otherwise. }
$$

Then $\operatorname{dim}_{\mathbf{C}} D=3$ and we can prove

Proposition. In the above notation, we have the following.

(i) $D_{\Xi}$ contains all nilpotent orbits.

(ii) Let $p \in \Gamma \backslash D_{\Xi}-\Gamma \backslash D$. Then, there exist an open neighborhood $U$ of $p$ in $\Gamma \backslash D_{\Xi}$ and an open set $\tilde{U}$ in $D_{\Xi}^{\sharp}$, in the topologies introduced in (6.1), which fit in the following commutative diagram:

$$
\begin{array}{ccccc}
\Gamma \backslash D_{\Xi} & \supset & U & \stackrel{\alpha}{\hookrightarrow} & \mathbf{C} \times S \\
\uparrow & & \uparrow & & \beta \uparrow \\
D_{\Xi}^{\sharp} & \supset & \tilde{U} & \hookrightarrow & \mathbf{C} \times \mathbf{R} \times \tilde{S}
\end{array}
$$

Here $\alpha: p \mapsto(0,0,0), \beta:(w, a, r, z) \mapsto\left(w, r e^{a i}, z\right)$. If we endow $\mathbf{C} \times S($ resp. $\mathbf{C} \times \mathbf{R} \times \tilde{S})$ with the product topology of the usual topology of $\mathbf{C}($ resp. $\mathbf{C} \times \mathbf{R})$ and $\mathcal{T}($ resp. $\tilde{\mathcal{T}})$, then both $\hookrightarrow$ are open immersions. 


\section{REFERENCES}

[AMRT] A. Ash, D. Mumford, M. Rapoport and Y. S. Tai, Smooth compactification of locally symmetric varieties, Math. Sci. Press, Brookline, 1975.

[BB] L. Baily and A. Borel, Compactifications of arithmetic quotients of bounded symmetric domains, Ann. of Math. 84 (1966), 442-528.

[BS] A. Borel and J.-P. Serre, Corners and arithmetic groups, Comment. Math. Helv. 48 (1973), $436-491$.

$[\mathrm{CCK}]$ J. A. Carlson, E. Cattani and A. Kaplan, Mixed Hodge structures and compactifications of Siegel's space, in: Journées de géométrie algébrique d'Angers 1979 (A. Beauville, ed.), Sijthohoff \& Noordhoff, 1980, pp. 77-105.

[CK] E. Cattani and A. Kaplan, Extension of period mappings for Hodge structures of weight 2, Duke Math. J. 44 (1977), 1-43.

[CKS] E. Cattani and A. Kaplan and W. Schmid, Degenerations of Hodge structures, Ann. of Math. 123 (1986), $457-535$.

[G] P. A. Griffiths, Periods of integrals on algebraic manifolds: Summary of main results and discussions of open problems, Bull. Amer. Math. Soc. 76 (1970), 228-296.

[HZ] M. Harris and S. Zucker, Boundary cohomology of Shimura varieties I. - Coherent cohomology on toroidal compactifications, Ann. scient. Éc. Norm. Sup. 27 (1994), 249-344.

[I] L. Illusie, Logarithmic spaces (according to K. Kato), in: Barsotti Symposium in Algebraic Geometry (V. Critstante and W. Messing, eds.), Perspectives in Math. 15, Academic Press, 1994, pp. 183-203.

[K1] K. Kato, Logarithmic structures of Fontaine-Illusie, in: Algebraic analysis, geometry, and number theory (J.-I Igusa, ed.), Perspectives in Math., Johns Hopkins University Press, Baltimore, 1989, pp. 191-224.

[K2] _ Toric singularity, Amer. J. Math. 116 (1994), 1073-1099.

[KkNc] K. Kato and C. Nakayama, Log Betti cohomology, log étale cohomology, and log de Rham cohomology of log schemes over C, Prepublications, Univ. Tokyo, UTMS 95-16 (1995).

[KyNy] Y. Kawamata and Y. Namikawa, Logarithmic deformations of normal crossing varieties and smoothing of degenerate Calabi-Yau varieties, Invent. Math. 118 (1994), 395-409.

[KU] K. Kato and S. Usui, Logarithmic Hodge structures and classifying spaces, in preparation.

[M] D. R. Morrison, Mirror symmetry and rational curves on quintic threefolds: A guide for mathematicians, J. Amer. Math. Soc. 6-1 (1993), 223-247.

[Sa] I. Satake, On compactification of the quotient spaces for arithmetically defined discontinuous groups, Ann. of Math. 72 (1960), 555-580.

[Sc] W. Schmid, Variation of Hodge structure; the singularities of the period mappings, Invent. Math. 22 (1973), $211-319$.

[U1] S. Usui, A numerical criterion for admissibility of semi-simple elements, Tôhoku Math. J. 45 (1993), $471-484$.

[U2] _ Complex structures on partial compactifications of arithmetic quotients of classifying spaces of Hodge structures, Tôhoku Math. J. 47 (1995), 405-429.

Graduate School of Mathematical Sciences, University of Tokyo, Komaba, Tokyo, 153, Japan

E-mail address: kkato@ms.u-tokyo.ac.jp

Graduate School of Science, Osaka University, Toyonaka, Osaka, 560, Japan

E-mail address: usui@math.wani.osaka-u.ac.jp 